\title{
Factores de riesgo de mortalidad en personas mayores de 65 años internadas en un hospital universitario, São Paulo, Brasil
}

\author{
Sandro J. Martins, ${ }^{1}$ Silvio L. Cardenuto ${ }^{1}$ y Valdir Golin ${ }^{1}$
}

RESUMEN Diversos estudios muestran que la edad en sí no es un factor independiente predictor de la supervivencia de las personas mayores gravemente enfermas, pero el grupo mayor de 65 años de edad no suele tener acceso a los recursos diagnósticos y terapéuticos más complejos. Con el continuo envejecimiento de la población se hace cada vez más importante poder determinar los factores de riesgo de mortalidad que afectan a las posibilidades de supervivencia de la gente mayor hospitalizada, especialmente en condiciones de urgencia. El objetivo de este trabajo fue analizar los factores de riesgo asociados con la mortalidad de personas mayores de 65 años internadas en el Servicio de Urgencia del hospital Santa Casa de São Paulo, institución académica de nivel terciario. El estudio se basó en un análisis por regresión logística no condicional de los datos personales recogidos en el hospital en las historias clinicas respectivas y otros documentos pertenecientes a los pacientes hospitalizados durante el período de julio de 1993 a marzo de 1994, inclusive. Durante dicho período se hospitalizó a 599 pacientes -326 hombres (54,4\%) y 273 mujeres (45,6\%) — con una mediana de edad de 73,3 años. Las razones principales de internamiento fueron neumonía $(14,4 \%)$, enfermedad cerebrovascular $(11,5 \%)$ e insuficiencia cardíaca $(8,2 \%)$. El total de defunciones ascendió a 160. Los pacientes que fallecieron tuvieron una estadía mediana en el hospital de 4 días (intervalo de 1 a 72), semejante a la de los que sobrevivieron (3 dias; intervalo de 0 a 35 días; $\mathrm{P}=0,29$ ). Según el análisis multivariado, independientemente del sexo, la edad, la raza, la observancia del tratamiento, el diagnóstico inicial y otros estados patológicos presentes, la hipertensión arterial (razón de posibilidades, $R P u$ odds ratio en inglés = 0,39, IC95\%: 0,23 a 0,68), la enfermedad pulmonar obstructiva crónica $(R P=0,45$; IC95\%: 0,22 a 0,95) y la diabetes mellitus ( $R P=0,50 ;$ IC95\%: 0,27 a 0,91) fueron factores predictores de supervivencia, mientras que la presencia de infecciones extrapulmonares $(R P=2,34$; IC95\%: 1,13 a 4,86) y el número de enfermedades preexistentes - una (RP = 2,78; IC95\%: 1,56 a 4,96), dos (RP = 4,56; IC95\%: 2,28 a 9,15) y más de dos (RP=15,88; IC95\%: 6,49 a 38,85) _ fueron factores de pronóstico independientes, indicadores de fallecimiento durante la hospitalización. Se concluye que el mejoramiento del diagnóstico y el tratamiento de las infecciones puede reducir la mortalidad de las personas de edad avanzada ingresadas en los servicios de urgencia. La multiplicidad de enfermedades, no la edad, fue el factor que aumentó el riesgo de muerte en este grupo de pacientes. No puede justificarse restringir el acceso de los pacientes geriátricos a los mejores recursos de diagnóstico y terapéutica, solo por la edad, si dichos recursos pueden resultar en mayor supervivencia, prevención de discapacidades o una mejor calidad de vida.

1 Hospital Santa Casa de São Paulo, Departamento de Medicina, Servicio de Urgencias. Toda la correspondencia referente a este artículo debe dirigirse a Sandro J. Martins, a la siguiente dirección: Av. Paulista 648 - apto. 2007, São Paulo, SP, Brasil, CEP 01310-100. Correo electrónico: sjm@mandic. com.br
Hoy en día la gente goza de una vida más larga, saludable, activa y productiva que en épocas anteriores. La supervivencia de un número cada vez mayor de personas de edad avan- zada contribuye al consumo de una elevada proporción de los recursos destinados a los servicios de salud, lo que a menudo plantea cuestiones éticas y sociológicas de difícil solución 
para la medicina moderna $(1,2)$. Por otra parte, la internación hospitalaria suele ser el primer paso en el camino hacia la institucionalización y pérdida de independencia de muchas personas de edad avanzada.

Pese a que los médicos tienden a no ofrecer a las personas mayores la oportunidad de tener acceso a los recursos diagnósticos y terapéuticos más complejos (3-6), diversos estudios han mostrado que la edad en sí no es un factor independiente predictor de la supervivencia a largo plazo de ancianos gravemente enfermos $(4,7,8)$. En consecuencia, actualmente reviste gran importancia para los médicos, los propios pacientes y toda la comunidad poder identificar los factores de riesgo de mortalidad que afectan a sus posibilidades de supervivencia durante la hospitalización, especialmente en condiciones de urgencia. El objetivo de este estudio fue evaluar la influencia de características demográficas y estados patológicos preexistentes en la mortalidad de las personas de edad avanzada internadas en los servicios de urgencia de un hospital universitario.

\section{MATERIALES Y MÉTODOS}

Se revisaron los registros de todos los pacientes de 65 o más años de edad internados en el Pronto Socorro Central, servicio de urgencias del hospital Santa Casa de São Paulo, durante el período de julio de 1993 a marzo de 1994, inclusive. En este sector de la institución se interna a los enfermos que necesitan cuidados inmediatos médicos o quirúrgicos no ortopédicos. De ordinario, cada paciente ingresado recibe, bajo supervisión apropiada, un examen médico completo realizado por un interno y un médico residente, el cual también redacta una historia clínica detallada del paciente. En esta se hacen constar los resultados de las pruebas iniciales de laboratorio (hemograma, mediciones bioquímicas y enzimáticas, urinálisis), telerradiografía del tórax y electrocardiograma con 12 derivaciones, así como los de pruebas más complejas (ultrasonografía, ecocardiografía, tomografía computadori- zada, centelleografía pulmonar, angiografía, estudios inmunohematológicos, radioinmunoensayos), de acuerdo con las necesidades que imponen las hipótesis diagnósticas formuladas.

Para este estudio se obtuvieron los datos demográficos de cada paciente (edad, sexo y raza), información sobre las enfermedades actuales y uso correspondiente de medicamentos, diagnóstico principal inicial, tiempo de estadía y condiciones del alta hospitalaria. Los diagnósticos establecidos se agruparon según los criterios de las listas especiales de tabulación para la mortalidad y la morbilidad de la Clasificación Estadística Internacional de Enfermedades y Problemas Relacionados con la Salud, décima revisión (9). Por su frecuencia y presunta importancia, los siguientes estados patológicos se marcaron para análisis cada vez que se encontraron presentes: hipertensión arterial sistémica; enfermedad pulmonar obstructiva crónica, diabetes mellitus, neoplasia, insuficiencia cardíaca, neumonía, y otros cuadros de infección y abdomen agudo.

La hipótesis de independencia entre frecuencias se evaluó con la prueba de Fisher (para 2 grados de libertad [GL]) y con la de ji cuadrado $\left(\chi^{2}\right)$ (si los GL son más de 2). Las distribuciones de valores se compararon por medio de la prueba de la U de Mann-Whitney. En todos los casos se utilizó un intervalo de confianza de $95 \%$ para la inferencia estadística. Con el fin de determinar los factores asociados con la mortalidad durante la hospitalización, los pacientes que fallecieron se compararon con los que sobrevivieron. Los datos obtenidos de este estudio se incorporaron en un modelo de regresión logística no condicional, con la muerte durante la hospitalización como variable dependiente. La asociación entre las características de los pacientes y la variable dependiente se estimó por medio de la razón de posibilidades (RP, odds ratio en inglés), obtenida por medio de dos coeficientes de regresión y expresada con un intervalo de confianza de 95\%. Los análisis estadísticos se llevaron a cabo con dos programas, Epi Info (versión 6.04) y MULTLR $(10,11)$.

\section{RESULTADOS}

Durante el período de estudio se hospitalizó a 599 pacientes mayores de 65 años. Más de la mitad (54,4\%) eran hombres y la mediana de edad del grupo fue 73,3 años. La mayoría de estos pacientes usaban regularmente algún medicamento prescrito para un estado patológico preexistente. Durante el período investigado fallecieron $160(26,7 \%)$ de los pacientes. El tiempo de estadía de estos pacientes en el hospital (mediana de 4 días con un intervalo de 1 a 72 días) fue semejante al de los pacientes que sobrevivieron (mediana de 3 días con un intervalo de 1 a 35 días; $P=0,29)$. El cuadro 1 resume las características generales de los pacientes. En el análisis univariado, no se mostró ninguna relación entre el sexo, la edad, la raza ni la observancia del tratamiento para las dolencias preexistentes y la mortalidad intrahospitalaria.

Las causas más comunes de internamiento fueron las enfermedades del sistema circulatorio, a las que correspondieron $41,4 \%$ de los diagnósticos principales de ingreso en el hospital (cuadro 2). Sin embargo, esas enfermedades representaron solamente $24,1 \%$ del total de fallecimientos. Entre las proporciones más altas de pacientes que fallecieron estaban 38,2 y 37,2\%, respectivamente, de los ingresados debido a lesiones, envenenamiento $y$ otras causas externas y por enfermedades del aparato respiratorio. La mortalidad fue menor $(11,8 \%)$ entre los ingresados por descompensación diabética. Se observó una relación directa, significativa y proporcional entre el número de dolencias concomitantes y el desenlace en muerte intrahospitalaria. Una vez ajustado según los datos demográficos, el número de enfermedades presentes y la observancia del tratamiento, ningún grupo de los diagnósticos de ingreso se asoció con una posibilidad mayor o menor de fallecimiento. Aun así, considerando la influencia de los demás factores estudiados, la presencia concomitante de una, dos y tres o más dolencias se asoció, respectivamente, con la posibilidad 2, 4 y 15 veces mayor de mortalidad. 
CUADRO 1. Características generales de las personas de edad avanzada ingresadas en el servicio de urgencias del hospital Santa Casa de São Paulo entre julio de 1993 y marzo de 1994, São Paulo, Brasil

\begin{tabular}{|c|c|c|c|c|c|c|}
\hline \multirow[b]{2}{*}{ Característica } & \multicolumn{2}{|c|}{ Casos (599) } & \multicolumn{2}{|c|}{ Fallecimientos (160) } & \multirow{2}{*}{$\begin{array}{c}\text { Mortalidad } \\
\%\end{array}$} & \multirow[b]{2}{*}{$P^{a}$} \\
\hline & $n$ & $\%$ & $n$ & $\%$ & & \\
\hline $\begin{array}{l}\text { Sexo } \\
\text { Masculino } \\
\text { Femenino }\end{array}$ & $\begin{array}{l}326 \\
273\end{array}$ & $\begin{array}{l}(54,4) \\
(45,6)\end{array}$ & $\begin{array}{l}94 \\
66\end{array}$ & $\begin{array}{l}(58,7) \\
(41,3)\end{array}$ & $\begin{array}{l}28,8 \\
24,2\end{array}$ & 0,23 \\
\hline $\begin{array}{l}\text { Edad (años) } \\
65-69 \\
70-74 \\
75-79 \\
\geq 80\end{array}$ & $\begin{array}{l}169 \\
150 \\
133 \\
147\end{array}$ & $\begin{array}{l}(28,2) \\
(25,0) \\
(22,2) \\
(24,6)\end{array}$ & $\begin{array}{l}38 \\
39 \\
43 \\
40\end{array}$ & $\begin{array}{l}(23,7) \\
(24,4) \\
(26,9) \\
(25,0)\end{array}$ & $\begin{array}{l}22,5 \\
26,0 \\
32,3 \\
27,2\end{array}$ & 0,29 \\
\hline $\begin{array}{l}\text { Raza } \\
\text { Caucásica } \\
\text { No caucásica }\end{array}$ & $\begin{array}{r}529 \\
70\end{array}$ & $\begin{array}{l}(88,3) \\
(11,7)\end{array}$ & $\begin{array}{r}142 \\
18\end{array}$ & $\begin{array}{l}(88,7) \\
(11,3)\end{array}$ & $\begin{array}{l}26,8 \\
25,7\end{array}$ & 0,95 \\
\hline $\begin{array}{l}\text { Uso de algún medicamento } \\
\text { No } \\
\text { Sí, uso regular } \\
\text { Sí, uso irregular } \\
\text { No sabe responder }\end{array}$ & $\begin{array}{r}26 \\
290 \\
98 \\
185\end{array}$ & $\begin{array}{r}(4,3) \\
(48,4) \\
(16,4) \\
(30,9)\end{array}$ & $\begin{array}{r}5 \\
20 \\
79 \\
56\end{array}$ & $\begin{array}{r}(3,1) \\
(12,5) \\
(49,4) \\
(35,0)\end{array}$ & $\begin{array}{l}19,2 \\
20,4 \\
27,2 \\
30,3\end{array}$ & 0,26 \\
\hline
\end{tabular}

a Prueba de Fisher con 2 grados de libertad (GL) o prueba de ji al cuadrado con más de 2 GL.

Al evaluar la relación entre la mortalidad y los principales factores de enfermedad concomitante identificados entre las personas incluidas en este estudio (cuadro 3), se observa que la hipertensión arterial sistémica y la diabetes mellitus fueron las enfermedades más frecuentemente halladas. La infección de las vías respiratorias bajas fue la tercera enfermedad más común y la que presentó mayor proporción de mortalidad $(40,0 \%)$. Considerando el efecto de las características demográficas, las enfermedades concomitantes, la observancia del tratamiento y los diagnósticos de ingreso, no fue malo el pronóstico de enfermos con hipertensión arterial sistémica, enfermedad pulmonar obstructiva crónica ni diabetes mellitus.
Se encontraron infecciones de las vías respiratorias bajas en 125 pacientes de los cuales fallecieron $50(40,0 \%)$. Estas enfermedades fueron más frecuentes entre los pacientes con más de 75 años de edad que entre los demás $(28,2 \%$ frente a $14,4 \%, P=0,0001)$. Se diagnosticaron infecciones extrapulmonares en 93 personas de las cuales fallecieron $36,6 \%$. En estos casos, las infecciones más comunes fueron las del sistema urinario $(29,0 \%)$, la erisipela y celulitis $(29,0 \%)$ y las infecciones intraabdominales $(14,0 \%) ; 10$ pacientes desarrollaron sepsis. Entre los enfermos con infecciones extrapulmonares hubo una mayor frecuencia de diabetes $(30,1$ frente a $19,4 \%, P=0,028)$ que entre los demás pacientes. En el análisis multivariado, la manifestación de alguna infección no pulmonar fue un factor independiente de mal pronóstico, que aumentó 2,34 veces la posibilidad de muerte durante la hospitalización.

Entre los pacientes ingresados por enfermedad del sistema circulatorio (cuadro 4), las causas principales de hospitalización fueron enfermedad cerebrovascular $(27,8 \%)$ e insuficiencia cardíaca (20,6\%). Con excepción de los pacientes ingresados por enfermedades de las venas y de los vasos y ganglios linfáticos (CIE 180-189) (incluidos los cuadros de várices esofágicas con hemorragia) en quienes la mortalidad

CUADRO 2. Diagnóstico inicial, enfermedad concomitante y mortalidad en 599 personas de edad avanzada ingresadas en el servicio de urgencias del hospital Santa Casa de São Paulo entre julio de 1993 y marzo de 1994, São Paulo, Brasil

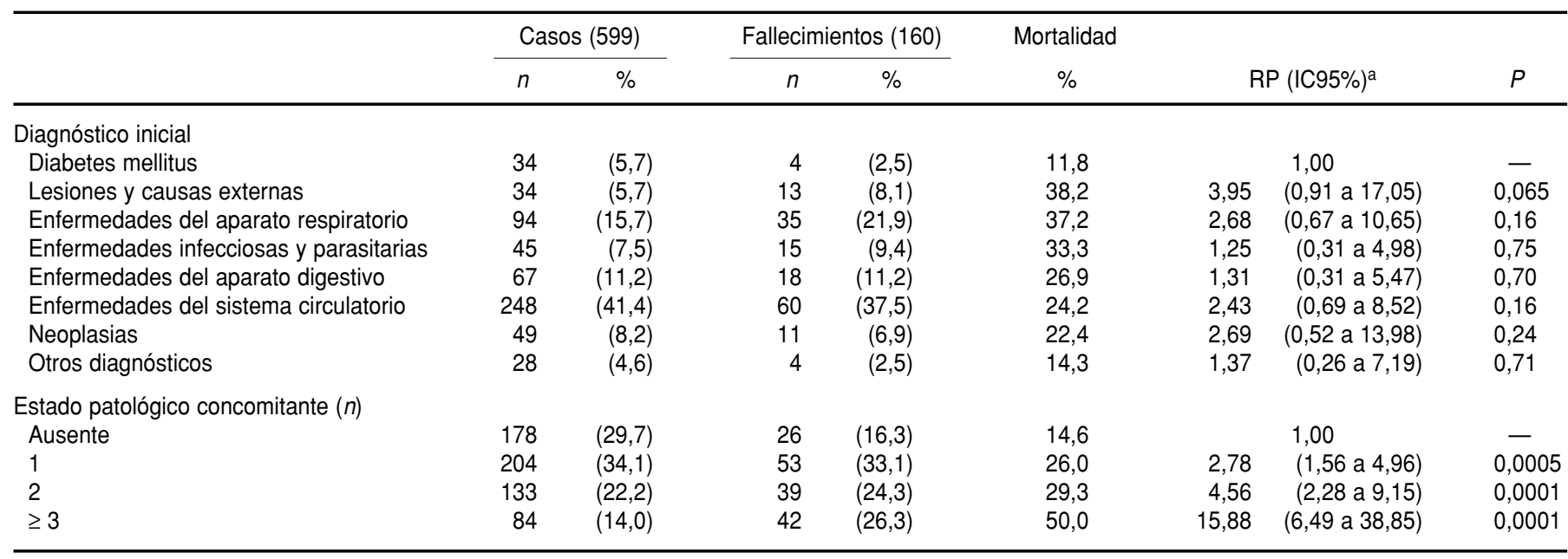

${ }^{a}$ Regresión logística: razón de posibilidades (RP, odds ratio en inglés) e intervalo de confianza de 95\% (IC95\%) para mortalidad ajustada por sexo, edad, raza, observancia del tratamiento para la enfermedad principal y estados patológicos concomitantes. 
CUADRO 3. Principales estados patológicos concomitantes y mortalidad asociada en personas de edad avanzada ingresadas en el servicio de urgencias del hospital Santa Casa de São Paulo entre julio de 1993 y marzo de 1994, São Paulo, Brasil

\begin{tabular}{lcccr}
\hline \multicolumn{1}{c}{ Estado patológico } & $\begin{array}{c}\text { Frecuencia } \\
\%\end{array}$ & $\begin{array}{c}\text { Mortalidad } \\
\%\end{array}$ & RP (IC95\%) & \multicolumn{1}{c}{$P$} \\
\hline Hipertensión arterial & 29,9 & 32,6 & $0,39(0,23$ a 0,68) & 0,0007 \\
Enfermedad pulmonar obstructiva crónica & 9,2 & 32,7 & $0,45(0,22$ a 0,95) & 0,035 \\
Diabetes mellitus & 21,0 & 24,6 & $0,50(0,27$ a 0,91) & 0,023 \\
Neoplasia & 12,5 & 26,7 & $0,94(0,38$ a 2,33$)$ & 0,89 \\
Insuficiencia cardíaca & 17,4 & 27,9 & $0,76(0,44$ a 1,31) & 0,32 \\
Infección de vías respiratorias bajas & 20,9 & 40,0 & $1,43(0,67$ a 3,03) & 0,35 \\
Infecciones extrapulmonares & 15,5 & 36,6 & $2,34(1,13$ a 4,86) & 0,022 \\
Abdomen agudo & 3,5 & 38,0 & $1,65(0,47$ a 5,82$)$ & 0,43 \\
\hline
\end{tabular}

a Regresión logística: razón de posibilidades (RP, odds ratio en inglés) e intervalo de confianza de 95\% (IC95\%) para mortalidad ajustada por sexo, edad, raza y observancia del tratamiento para la enfermedad principal al ingreso.

CUADRO 4. Enfermedades del sistema circulatorio como causa de ingreso de 248 personas de edad avanzada en el servicio de urgencias del hospital Santa Casa de São Paulo, julio de 1993 a marzo de 1994, São Paulo, Brasil

\begin{tabular}{|c|c|c|c|c|c|}
\hline \multirow[b]{2}{*}{ Enfermedad } & \multicolumn{2}{|c|}{ Casos (248) } & \multicolumn{2}{|c|}{ Fallecimientos $(60)$} & \multirow{2}{*}{$\begin{array}{c}\text { Mortalidac } \\
\%\end{array}$} \\
\hline & $n$ & $\%$ & $n$ & $\%$ & \\
\hline Enfermedad cerebrovascular & 69 & $(27,8)$ & 17 & $(28,3)$ & 24,6 \\
\hline Insuficiencia cardíaca & 51 & $(20,6)$ & 14 & $(23,3)$ & 27,5 \\
\hline Enfermedad cardíaca isquémica & 40 & $(16,1)$ & 10 & $(16,7)$ & 25,0 \\
\hline Enfermedad arterial & 34 & $(13,7)$ & 6 & $(10,0)$ & 17,6 \\
\hline Enfermedad hipertensiva & 18 & $(7,3)$ & 3 & $(5,0)$ & 16,7 \\
\hline Enfermedad de las venas & 14 & $(5,6)$ & 7 & $(11,7)$ & 50,0 \\
\hline Otra enfermedad cardiovascular & 22 & $(8,9)$ & 3 & $(5,0)$ & 13,6 \\
\hline
\end{tabular}

llegó a 50\%, los pacientes ingresados por insuficiencia cardíaca, enfermedad cerebrovascular y enfermedades cardíacas isquémicas fueron los que murieron en mayor proporción. En el análisis multivariado esos subgrupos de diagnósticos no se relacionaron significativamente con una mayor o menor mortalidad intrahospitalaria.

\section{DISCUSIÓN}

Los resultados de este estudio incrementan los conocimientos existentes en nuestro medio con respecto al pronóstico de un importante segmento de pacientes geriátricos en los hospitales públicos del Brasil. Se trata de los pacientes no institucionalizados ingresados para tratamiento médico en condiciones de urgencia. forma habitual de atención que reciben las personas de edad avanzada en los hospitales públicos del Brasil.

Tal como se ha observado en otros estudios $(2,19)$, las enfermedades del sistema circulatorio fueron las causas más comunes de hospitalización y de enfermedad preexistente (1). Pese a que la hipertensión arterial sistémica constituye en general para la población de edad avanzada un importante factor de riesgo de mortalidad por todas las causas (19), aquí no se relacionó con una mayor posibilidad de mortalidad intrahospitalaria. Según han observado también otros autores (19-21), la disponibilidad actual de productos farmacológicos eficaces y seguros para el tratamiento de la hipertensión arterial ha beneficiado sobre manera a las personas mayores hospitalizadas para controlar y estabilizar los niveles de tensión arterial en emergencias hipertensivas, y es capaz de reducir el riesgo de complicaciones y muerte por enfermedades cardiovasculares. Del mismo modo, el tratamiento adecuado de las personas de edad avanzada hospitalizadas debido a enfermedad obstructiva crónica o diabetes mellitus permite con cada vez mayor frecuencia la recuperación de estos pacientes (22-24).

En relación con el diagnóstico de ingreso, la mayor proporción de mortalidad correspondió a los pacientes internados por lesiones y causas externas $(38,2 \%)$, cifra algo más alta que las que se encuentran en la literatura, las cuales varían de 15 a 30\% (25-27). La mayor parte de estos pacientes $(88,2 \%)$ habían sufrido traumas. La limitada reserva fisiológica del paciente geriátrico y las dolencias preexistentes justifican la mortalidad observada $(25,26$, 28). Cabe resaltar que no se incluyeron en el estudio muchos pacientes de edad avanzada con lesiones ortopédicas provocadas por caídas accidentales, que fueron atendidos en otros sectores del hospital durante el mismo período de estudio.

La elevada mortalidad por infecciones de las vías respiratorias bajas (40\%) así como su relación con la edad se aproximan a lo que han descrito otros autores $(1,2,19,28,29)$. A la luz 
de los conocimientos actuales se espera que la inmunización de las personas de edad avanzada contra los virus de la influenza y los neumococos pueda disminuir la incidencia y la gravedad de estas infecciones y prevenga hasta $50 \%$ de los fallecimientos intrahospitalarios por enfermedades del sistema respiratorio (30-33).

La mortalidad por infecciones no pulmonares en personas de edad avanzada se ha estimado entre $20 \mathrm{y}$ $40 \%$ (34-37); en este estudio fallecieron $36,7 \%$ de los pacientes con infecciones que no afectaban al sistema respiratorio. En vista de todos los factores estudiados, la posibilidad de que esos pacientes murieran durante el período de hospitalización fue 2,34 veces mayor que los que tenían otras enfermedades.

Hay diversos factores que contribuyen a aumentar la incidencia y la letalidad de las infecciones en las personas de edad avanzada: alteraciones del sistema inmunitario, factores externos al sistema inmunitario, alteraciones anatomofisiológicas propias del envejecimiento y demoras de diagnóstico. Sobre la base de los resultados obtenidos en este estudio, el mejoramiento del diagnóstico y el tratamiento de las infecciones se destaca como la intervención médico-hospitalaria potencialmente más importante para redu- cir la mortalidad de las personas de edad avanzada ingresadas en los servicios de urgencia.

La demora al diagnosticar las infecciones es un problema clínico importante y un factor preponderante en la morbilidad y la mortalidad de la gente mayor. Esto suele ocurrir con las infecciones intraabdominales como la colecistitis, la apendicitis e infecciones secundarias a la perforación del colon por cáncer o enfermedad diverticular, las cuales siguen un curso atípico con señales externas más bien leves (27, 36). La tuberculosis, la neumonía bacteriana, la endocarditis, la bacteriemia y las meningoencefalitis tienen también una evolución clínica atípica y pueden presentarse solo como fatiga, estados de confusión agudos, caídas frecuentes y fiebre de origen indeterminado, lo que plantea dificultades de diagnóstico considerables $(37,38)$. El envejecimiento representa un proceso fisiológico único y complejo, en el cual hay una gradual interacción del efecto concomitante de las modificaciones del organismo y de las enfermedades adquiridas, que debilita paulatinamente a la persona. Intuitivamente, parece lógico que las personas con múltiples problemas médicos evolucionen de forma menos favorable cuando adquieren nuevas enfermeda- des. Este hecho se destacó nítidamente en este estudio, que demostró la influencia directa, proporcional e independiente de la presencia de enfermedades concomitantes en la mortalidad intrahospitalaria de gente mayor hospitalizada por urgencias medicoquirúrgicas. Los pacientes con tres o más enfermedades preexistentes en relación con la causa del ingreso hospitalario tuvieron 15 veces más posibilidades de fallecer durante su estadía. Como el número de enfermedades coexistentes tiende a aumentar con la edad, es razonable suponer que, en muchos estudios donde no se controla la influencia de este factor, una importante fracción del exceso del riesgo de muerte asociado con la edad se debe principalmente a la multiplicidad de enfermedades del paciente geriátrico.

Cada vez se observa más que la edad avanzada en sí no constituye un factor de mal pronóstico en diversas situaciones médicas. De acuerdo con nuestros conocimientos actuales, no puede justificarse restringir el acceso del paciente geriátrico a los mejores recursos de diagnóstico y terapéutica disponibles solo porque ha envejecido, siempre que dichos recursos puedan resultar en una mayor supervivencia, la prevención de disfunciones o una mejor calidad de vida

\section{REFERENCIAS}

1. Gorzoni ML, Lima CA da C. Análise dos parâmetros clínicos de idosos internados em enfermaria de clínica médica. Rev Assoc Med Brasil 1995;41:227-232.

2. Ramos LR, Saad PM. Morbidade da população idosa. En: Fundação Sistema Estadual de Análise de Dados. O idoso na grande São Paulo. São Paulo: SEADE; 1990. Pp. 161-172.

3. Sesso R, Fernandes PF, Anção M, Drummond M, Draibe S, Sigulem D, et al. Acesso ao tratamento dialítico crônico: deficiente e desigual. Rev Assoc Med Brasil 1996;42:84-88.

4. Chelluri L, Pinsky MR, Donahoe MP, Grenvik A. Long-term outcome of critically ill elderly patients requiring intensive care. JAMA 1993;269:3119-3123.

5. Tresch DD, Brady WJ, Aufderheide TP, Lawrence SW, Williams KJ. Comparison of elderly and younger patients with out-of-hospital chest pain. Arch Intern Med 1996;156: 1089-1093.
6. Bearden D, Allman R, McDonald R, Miller S, Pressel S, Petrovich H. Age, race, and gender variation in the utilization of coronary artery bypass surgery and angioplasty in SHEP. J Am Geriatr Soc 1994;42:1143-1149.

7. Suárez G, Herrera M, Vera A, Torrado E, Ferris J, Arboleda JA. Prediction on admission of in-hospital mortality in patients older than 70 years with acute myocardial infarction. Chest 1995;198:83-88.

8. Peterson ED, Jollis JG, Bebchuk JD, De Long ER, Muhlbaier LH, Mark DB, et al. Changes in mortality after myocardial revascularization in the elderly: the national Medicare experience. Ann Intern Med 1994;121: 919-927.

9. Organización Panamericana de la Salud. Clasificación Estadística Internacional de Enfermedades y Problemas Relacionados con la Salud. 10a rev. Washington, DC: OPS; 1995. (Publicación científica 554).
10. Campos-Filho N, Franco EL. A micro computer program for logistic regression by unconditional and conditional maximum likelihood methods. Am J Epidemiol 1989;129: 439-444.

11. Dean AG, Dean JA, Coulombier D, Burton $\mathrm{AH}$, Brendel KA, Smith DC, et al. Epi Info, Version 6: a word processing, database, and statistic program for epidemiology on microcomputers. Stone Mountain: USD Inc; 1994. Pp. 150-168.

12. Young M, Marrie TJ. Interobserver variability in the interpretation of chest roentgenograms of patients with possible pneumonia. Arch Intern Med 1994;154:2729-2732.

13. Gudmundsson A, Carnes M. Geriatric assessment: making it work in primary care practice. Geriatrics 1996;51:55-65.

14. Tsang P, Severs MP. A study of appropriateness of acute geriatric admissions and an assessment of the Appropriateness Evaluation Protocol. J R Coll Physicians 1995;29:311-314. 
15. Kothari RU, Brott T, Broderick JP, Hamilton CA. Emergency physicians: accuracy in the diagnosis of stroke. Stroke 1995;26:2238-2241.

16. Corti MC, Guralnik JM, Salive ME, Sorkin JD. Serum albumin level and physical disability as predictors of mortality in older persons. JAMA 1994;272:1036-1042.

17. Constans T, Bacq Y, Brechot JF, Guilmot JL, Choutet $\mathrm{P}$, Lamisse F. Protein-energy malnutrition in elderly medical patients. J Am Geriatr Soc 1992;40:263-268.

18. Terzian C, Frye EB, Piotrowski ZH. Admission hyponatremia in the elderly: factors influencing prognosis. J Gen Intern Med 1994;9: 89-91.

19. Glynn RJ, Field TS, Rosner B, Hebert PR, Taylor JO, Hennekens $\mathrm{CH}$. Evidence for a positive linear relation between blood pressure and mortality in elderly people. Lancet 1995;345: 825-829.

20. Glynn JR, Brock DB, Harris T, Havlik RJ, Chrischilles EA, Ostfeld AM, et al. Use of antihypertenstive drugs and trends in blood pressure in the elderly. Arch Intern Med 1995;155:1855-1860.

21. Gorelick PB. Stroke prevention. Arch Neurol 1995;52:347-355.

22. Chang JT, Moran MB, Cugell DW, Webster JR. COPD in the elderly-a reversible cause of functional impairment. Chest 1995;108:736-740.

23. Nasri F, de Sá JR, Schimidt MLR. Hipoglicemiantes orais e insulinas. En: Gorzoni ML,
Neto JT, eds. Terapêutica clínica no idoso. São Paulo: Sarvier/APM;1995. Pp.148-160.

24. Ball P. Epidemiology and treatment of chronic bronchitis and its exacerbations. Chest 1995; 108(supl):43-52.

25. Zietlow SP, Capizzi PJ, Bannon MP, Farnell MB. Multisystem geriatric trauma. J Trauma 1994;37:985-988.

26. Rasslan S, Saad Jr R. Peritonitis. En: Rasslan S, ed. Afecções cirúrgicas de urgência. São Paulo: Robe Ed; 1995. Pp. 25-46.

27. Villar JIB, Lázaro MAG, Sancho CA, Peracho $\mathrm{CV}$. Incidencia anual y mortalidad asociada a las infecciones intrahospitalarias del tracto respiratorio inferior en un hospital comarcal. Rev Clin Esp 1994;194:282-287.

28. Schwab CW, Kauder DR. Trauma in geriatric patient. Arch Surg 1992;127:701-706.

29. Brown RB. Community-acquired pneumonia: diagnosis and therapy of older adults. Geriatrics 1993;48:43-50.

30. Gross PA, Hermogenes AW, Sacks HS, Lau J, Levandowski RA. The efficacy of influenza vaccine in elderly persons. Ann Intern Med 1995;123:518-527.

31. Fine MJ, Smith MA, Carson CA, Meffe F, Sankey SS, Weisfeld LA, et al. Efficacy of pneumoccoccal vaccination in adults: a metaanalysis of randomized controlled trials. Arch Intern Med 1994;154:2666-2677.

32. Fiebach N, Beckett W. Prevention of respiratory infections in adults: influenza and pneu- mococcal vaccines. Arch Intern Med 1994;154: 2545-2557.

33. Hedlund JU, Kalin ME, Ortqvist AB, Henrichsen J. Antibody response to pneumococcal vaccine in middle-aged and elderly patients recently treated for pneumonia. Arch Intern Med 1994;154:1961-1965.

34. Louria DB, Sen P, Sherer CB, Farrer WE. Infections in older patients: a systematic clinical approach. Geriatrics 1993;48:28-34.

35. Moulias R. Âge et immunité. Biomed Pharmacother 1987;41:115-121.

36. Kettunen J, Paajanen H, Kostiainen S. Emergency abdominal surgery in the elderly. Hepatogastroenterology 1995;42:106-108.

37. Werner GS, Schultz R, Fuchs JB. Infective endocarditis in the elderly in the era of transesophageal echocardiography: clinical features and prognosis compared with younger patients. Am J Med 1996:100:90-97.

38. Chassagne P, Perol MB, Doucet J, Trivalle C, Menard JF, Manchon ND, et al. Is presentation of bacteremia in the elderly the same as in younger patients? Am J Med 1996;100:65-70.

Manuscrito recibido el 3 de septiembre de 1997 y aceptado para publicación en versión revisada el 2 de marzo de 1999
ABSTRACT

Mortality risk factors for persons over age 65 hospitalized in a university hospital in São Paulo, Brazil
Various studies show that age in itself is not an independent predictive factor for the survival of critically ill older persons. In spite of that fact, people over 65 tend not to have access to the most-sophisticated diagnostic and therapeutic resources. With the continued aging of the population, it is increasingly important to be able to determine the mortality risk factors that affect the survival possibilities of hospitalized older people, especially in emergency situations. The objective of this study was to analyze the risk factors related to mortality among people over 65 admitted to the emergency service of the Santa Casa Hospital of São Paulo, a third-level academic institution. The study was based on a nonconditional logistic regression analysis of personal data in the clinical histories and other documents of the hospitalized patients. From July 1993 through March 1994, 599 patients over 65 were hospitalized. They included 326 men $(54.4 \%)$ and 273 women $(45.6 \%)$, with a median age of 73.3 years. The primary reasons for admission were pneumonia $(14.4 \%)$, cerebrovascular disease $(11.5 \%)$, and congestive heart failure $(8.2 \%)$. There were 160 deaths among the group. Those who died had a median stay in the hospital of 4 days (range, 1 to 72), which was similar to that of those who survived (median, 3 days; range, 0 to 35 days; $P=0.29$ ). According to multivariate analysis, factors predictive of survival were hypertension (odds ratio $=0.39$ 95\% CI: 0.23 to 0.68 ), chronic obstructive pulmonary disease (OR $=0.45 ; 95 \%$ CI: 0.22 to 0.95 ), and diabetes mellitus ( $\mathrm{OR}=0.50 ; 95 \% \mathrm{CI}: 0.27$ to 0.91$)$. This was true regardless of sex, age, race, compliance with treatment, initial diagnosis, and other pathological conditions present. On the other hand, two factors were predictive of mortality during hospitalization. They were the presence of extrapulmonary infections $(\mathrm{OR}=$ 2.34; 95\% CI: 1.13 to 4.86$)$ and the number of preexisting illnesses: one (OR $=2.78 ; 95 \%$ CI: 1.56 to 4.96$)$, two (OR $=4.56 ; 95 \% \mathrm{CI}: 2.28$ to 9.15$)$, and three or more $(\mathrm{OR}=15.88$; 95\% CI: 6.49 to 38.85 ). This study shows that improving the diagnosis and treatment of infections can reduce mortality of elderly persons admitted to emergency services. Rather than age, the multiplicity of diseases was the factor that increased the risk of death among these patients. There is no justification for using age alone to limit the access that older patients have to better diagnosis and treatment resources, if these resources can result in better survival rates, fewer disabilities, or a better quality of life. 